\section{Putting DNA in its place}

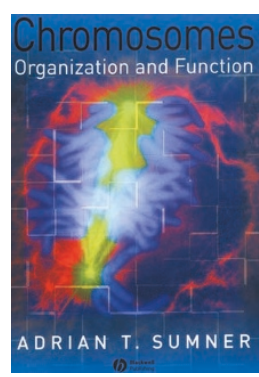

\section{Chromosomes: Organization and Function}

\author{
by Adrian T. Sumner
}

Blackwell Science $\boldsymbol{\bullet} 2003$

Price: $\$ 69.95 / £ 29.50$

\section{Wallace F. Marshall}

Ask a pig farmer to write a book about agriculture and you will end up reading a lot about how to raise pigs, but not much about harvesting wheat. By the same token, because most books about chromosomes are written by molecular biologists, they tend to focus almost entirely on DNA, and coverage of larger-scale issues such as chromosomes and nuclei tends to be given short shrift. And yet, the broader cell biological context within which DNA functions and duplicates cannot be ignored. The importance of the cell cycle, mitosis and nuclear architecture are beyond dispute. What is needed, therefore, is a book that can span the molecular and cellular aspects of chromosomes, allowing cell biologists to assimilate the explosion of molecular information while allowing molecular biologists to benefit from the insights yielded by cell-biological approaches. Chromosomes: Organization and Function by Adrian Sumner is the book that we have all been waiting for.

Even a casual glance at the table of contents will reveal the unique nature of this book. The range of topics covered is impressive. Of course, the standard topics, such as DNA replication and transcriptionare, are there. But unlike many books, these subjects do not dominate to the exclusion of all else. Instead, a wide range of aspects of chromosome biology are all given equal weight, and I simply cannot think of any other text that treats a comparable range of topics. Every major part or feature of chromosomes is covered in its own chapter; for example, telomeres have a chapter unto themselves, as do the nucleolus, sex chromosomes and euchromatin. The only standard topic that I had expected to see that was not treated was DNA topology. Specialized topics are treated similarly well; for example, there is an entire chapter on polytene chromosomes and another on lampbrush chromosomes. Other unusual topics covered include the chromosome changes that accompany evolution, the organization of chromosomes in interphase, and artificial chromosomes. I was particularly impressed by the chapter on chromosomes and disease, which ties together a surprisingly wide range of dis-

Wallace F. Marshall is in the Department of Molecular, Cellular and Developmental Biology, Yale University, New Haven, CT 06520, USA. e-mail: wallace.marshall@yale.edu eases, such as cancer and Bloom's Syndrome, by showing their underlying basis in chromosome defects. By interposing the most mainstream molecular studies, such as histone acetylation, with the most arcane cytology, such as that done on lampbrush chromosomes, Sumner weaves together a range of different types of information into a cohesive whole.

A major key to the success of this book is that it is written at precisely the right level of detail, outlining the major molecular players involved in each process without becoming bogged down in the nitty-gritty mechanistic details. A great example is the coverage of mitosis, where the book describes not just the physical processes of chromosome condensation, alignment on the metaphase plate and so forth, but also the molecules involved in sister chromatid cohesion, spindle assembly and kinetochore function. To get down to this level of practical detail, without going overboard, is tricky, but Sumner pulls it off perfectly by selecting just the most important details for presentation and giving the reader a taste of various aspects of chromosome biology without an overabundance of detail.

In addition to its superbly balanced organization, this book is also well-written. Sumner's prose takes a question-driven approach, asking, for example, "what does the nucleolus do?" or "what have we learnt from lampbrush chromosomes?” This makes for easy reading and also flags the key points for the reader. Another feature that makes the text easier to read is the extensive use of illustrations. The diagrams and micrographs convey the visual appeal that has sucked so many of us into careers in cell biology, but they also are used in a careful way to illustrate the points that the text is making. There is nothing gratuitous in this book.

Another notable feature of this book is the way it balances old and current literature. Chromosome research has an extremely long history, and the old literature is full of useful information that has largely been overlooked in recent decades. Sumner does an excellent job of extracting useful content from the old literature without missing a beat in his coverage of the most recent work. The current references (and the text is full of them) run up to 2001, which is good considering the time-line of book publication. The effort to make the book up to date was, however, taken a little bit too far by the inclusion of web links at the end of some chapters. The idea, of course, is to allow readers to access the most recent information on the web, but given the speed with which web addresses change or become broken, it is likely that many of these links will not work in the near future, making the whole exercise a bit futile.

This book would be perfect for anyone beginning to work on chromosomes who wants to get a rapid overview of the field. I also recommend this book to established chromosome biologists, simply because it is edifying to see the range of topics covered in this book knitted together so skillfully. Moreover, the book is full of interesting details that ensure its utility, even for experts. The required background knowledge for this book is not extensive, making it suitable for an upper level undergraduate or graduate course on chromosome biology or as a supplementary text for an advanced molecular biology course. 